\title{
Value of high-frequency ultrasound \\ biomicroscopy in uveitis

\section{Abstract}

Purpose To determine the use of highfrequency ultrasound biomicroscopy (UBM) in the assessment of inflammatory lesions of the iris, ciliary body, pars plana and peripheral vitreous, and in particular to determine the proportion of cases for which UBM contributed significant additional, hitherto inaccessible, information.

Methods Charts of patients seen in the uveitis clinic at University Eye Hospital from November 1994 to September 1999 for whom a UBM investigation had been performed were analysed. UBM was performed in a standard manner, using a Humphrey UBM 840 system. The clinical relevance of the UBM findings was determined for the whole series and for the following six subgroups of patients arbitrarily established according to the type and location of pathology: hypotony, pseudophakic uveitis, iris and ciliary body pathology excluding hypotony, pars plana pathology, scleritis and Toxocara uveitis. Findings were classified as positive when they confirmed a suspected diagnosis of lesional process or when they gave essential information. Findings were classified as essential when they led to the diagnosis or when they modified therapeutic intervention. Results During the study period 111 eyes of 77 patients were included. UBM findings contributed essential information that allowed a diagnosis to be reached or that influenced treatment in $43 \%$ of cases. It yielded positive findings in $91 \%$ of cases, enabling assessment of morphological changes in the iris, ciliary body, and retroiridal and peripheral vitreous induced by intraocular inflammatory or pseudo-inflammatory disorders. Specific UBM signs, present in all patients, were identified in Toxocara uveitis. The groups of patients that benefited most from UBM examination were those with hypotony $(83 \%$ essential findings) and opaque media (100\% essential findings).

Conclusion For uveitis patients with an inflammatory process situated in the iris/ ciliary body/pars plana/retroiridal vitreous areas, UBM was of great clinical value and improved the management in a significant manner.
Key words Ultrasound biomicroscopy, Uveitis

Evaluation of the inflammatory involvement of the iris stroma and retroiridal face, ciliary body, pars plana and retroiridal vitreous is sometimes important. These structures are not readily accessible with routine examination methods. Evaluation of the retrolenticular and retroiridal space is even more important when no visual access to the posterior segment is possible because of opaque media. In such cases ultrasonography is the method of choice. B-scan ultrasonography has become an essential and well-established device to help diagnose and manage ocular and orbital disorders. In recent years high-frequency ultrasonography has been introduced and made available to clinical practice. ${ }^{1,2}$ The method has been named ultrasound biomicroscopy (UBM) and is based on high-frequency transducers incorporated into a B-mode clinical scanner. This technology allows quasi-histological sections up to 3-6 $\mathrm{mm}$ in depth to be obtained in vivo, giving access to structures in the anterior part of the posterior segment that cannot be visualised otherwise or that are inaccessible because of opaque media. The method has been shown to be valuable in non-inflammatory pathologies of the anterior segment the ciliary body and the retoiridal space. $^{3-6}$

We investigated the role of UBM in a group of selected patients suffering from uveitis in which information was needed regarding the above-named structures. We determined in particular the proportion of cases for which UBM contributed significant additional, hitherto inaccessible, information.

\section{Patients and methods}

Charts from patients seen from November 1994 to July 1999 (57 months) in the uveitis unit of the University Eye Hospital for whom a UBM examination had been performed were reviewed. Patients were classified according to the anatomical location of the suspected lesion and according to the specific pathology, present or suspected, that constituted the indication to perform a UBM examination. These two parameters were used to establish an arbitrary subdivision of patients into six groups of

\section{V.T. Tran}

C.P. Herbort

Inflammatory Eye Diseases La Source Eye Center

Lausanne, Switzerland

P. LeHoang

Department of

Ophthalmology

Hôpital de La Pitié-

Salpétrière

Paris, France

\section{C.P. Herbort}

University of Lausanne Lausanne, Switzerland

Carl P. Herbort, MD, PD Inflammatory Eye Diseases La Source Eye Center

2, Ave des Bergières $\mathrm{CH}-1004$ Lausanne

Switzerland

Received: 7 January 2000 Accepted in revised form: 18 May 2000 


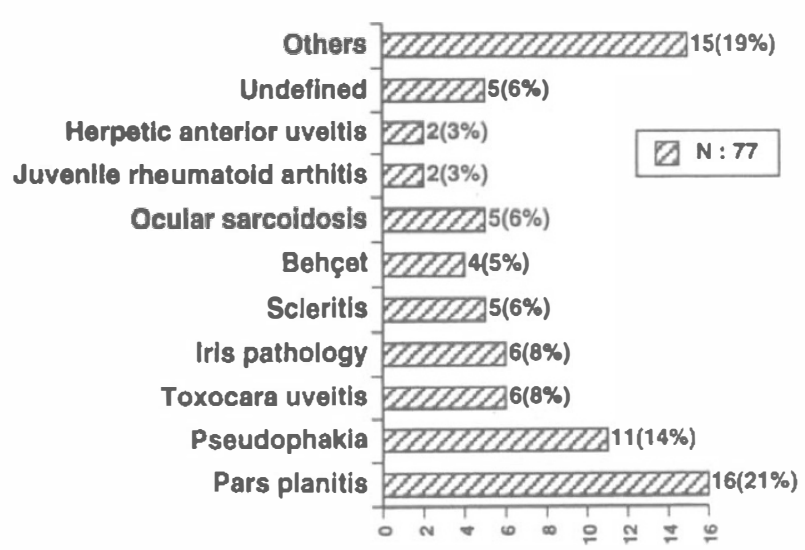

Fig. 1. Distribution of specific diagnoses in a total of 77 patients. 'Others' includes the following conditions: hypotony due to traumatic cyclodialysis (2), hypotony due to atrophy of ciliary body (2), Vogt-Koyanagi-Harada uveitis (2), cyclodialysis of unknown origin (1), uveal effusion syndrome (1), luxated lens (1), peripheral retinal mass in a case of Eales' disease (1), irido-corneal endothelial syndrome (1), Candida vitreitis (1), syphilitic uveitis (1), isolated ciliary body cyst (1), ciliary body metastasis (1).

pathologies: (1) hypotony, (2) pseudophakic uveitis, (3) iris and ciliary body pathology excluding hypotony, (4) pars plana pathology, (5) scleritis and (6) Toxocara uveitis. Additionally, patients already included in the above-named groups for whom UBM was performed because of opaque media, were re-analysed separately.

The proportion of cases among all uveitis patients seen during the period that needed UBM examination was calculated, and the proportion of cases in which UBM contributed essential information to help establish a diagnosis or to guide the management was determined. UBM findings were defined as essential when the information obtained helped a diagnosis to be reached or influenced the therapeutic attitude. A typical example of this situation would be a patient with hypotony for whom UBM identified a tractional ciliary body detachment due to a cyclitic membrane, indicating that surgical intervention would be recommended. UBM findings were defined as positive when they were essential or when they confirmed a suspected diagnosis or lesional process without significantly influencing the therapeutic attitude. A typical example of this situation would be the identification of condensations and deposits at the level of the pars plana in a case of intermediate uveitis, confirming the diagnosis of pars planitis. In all other cases UBM findings were defined as negative or non-contributory.

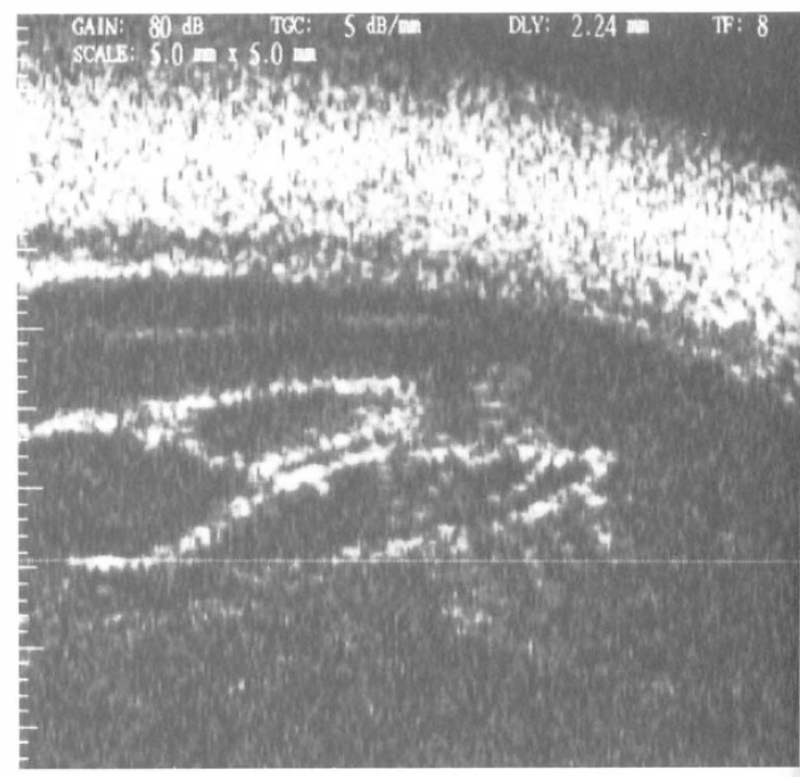

Fig. 2. Toxocara uveitis. UBM shows the typical pseudocystic aspect of the vitreous.

UBM was performed using a Humphrey UBM 840 highfrequency, high-resolution system (Humphrey Instruments, San Leandro, CA). Topical anaesthesia was obtained by applying several drops of $1 \%$ tetracaine. A $21 \mathrm{~mm}$ diameter cylindric eye cup was inserted between the eyelids and placed on the eyeball. Sterile physiological salt solution or methylcellulose solution was used to fill the eye cup. Hourly radial and longitudinal scans were performed over $360^{\circ}$ showing the irido-corneal angle, the ciliary body and the pars plana areas.

\section{Results}

During the study period 111 eyes of 77 patients were analysed by UBM. The mean age of the patients was $34.6 \pm 22.7$ years (range $5-84$ years). Thirty-six were male and 41 were female. During this period a total number of 612 patients were seen in the uveitis unit. Thus the proportion of patients for whom a UBM was performed was $12.5 \%(77 / 612)$ patients. This represents, however, a slight overestimation of the proportion of patients in whom UBM is indicated, as 12 of $77(16 \%)$ patients were specifically sent for a UBM examination, mostly from the hospital La Pitié-Salpétrière, Paris, France, because the instrument used is not licensed in France.

Table 1. Classification of UBM data by pathological categories

\begin{tabular}{lcccl}
\hline Pathology & No. $(\%)$ of patients & Essential UBM data & Positive UBM data & Non-contributory UBM data \\
\hline 1. Toxocara uveitis & $6(8 \%)$ & $6 / 6(100 \%)$ & $6 / 6(100 \%)$ & 0 \\
2. Hypotony & $12(16 \%)$ & $10 / 12(93 \%)$ & $12 / 12(100 \%)$ & 0 \\
3. Pseudophakic uveitis & $11(14 \%)$ & $4 / 11(36 \%)$ & $10 / 11(91 \%)$ & $1 / 11(9 \%)$ \\
4. Iris and ciliary body pathology & $17(22 \%)$ & $5 / 17(29 \%)$ & $14 / 17(82 \%)$ & $3 / 17(18 \%)$ \\
$\quad($ excluding hypotony) & & & & \\
5. Pars plana pathology & $26(33 \%)$ & $7 / 26(27 \%)$ & $23 / 26(88 \%)$ & $3 / 26(12 \%)$ \\
6. Scleritis & $5(6 \%)$ & 0 & $5 / 5(100 \%)$ & 0 \\
7. Total & 77 & $32 / 77(42 \%)$ & $70 / 77(91 \%)$ & $7 / 77$ \\
8. Opaque media & 9 & $9 / 9(100 \%)$ & 0 & 0 \\
\hline
\end{tabular}



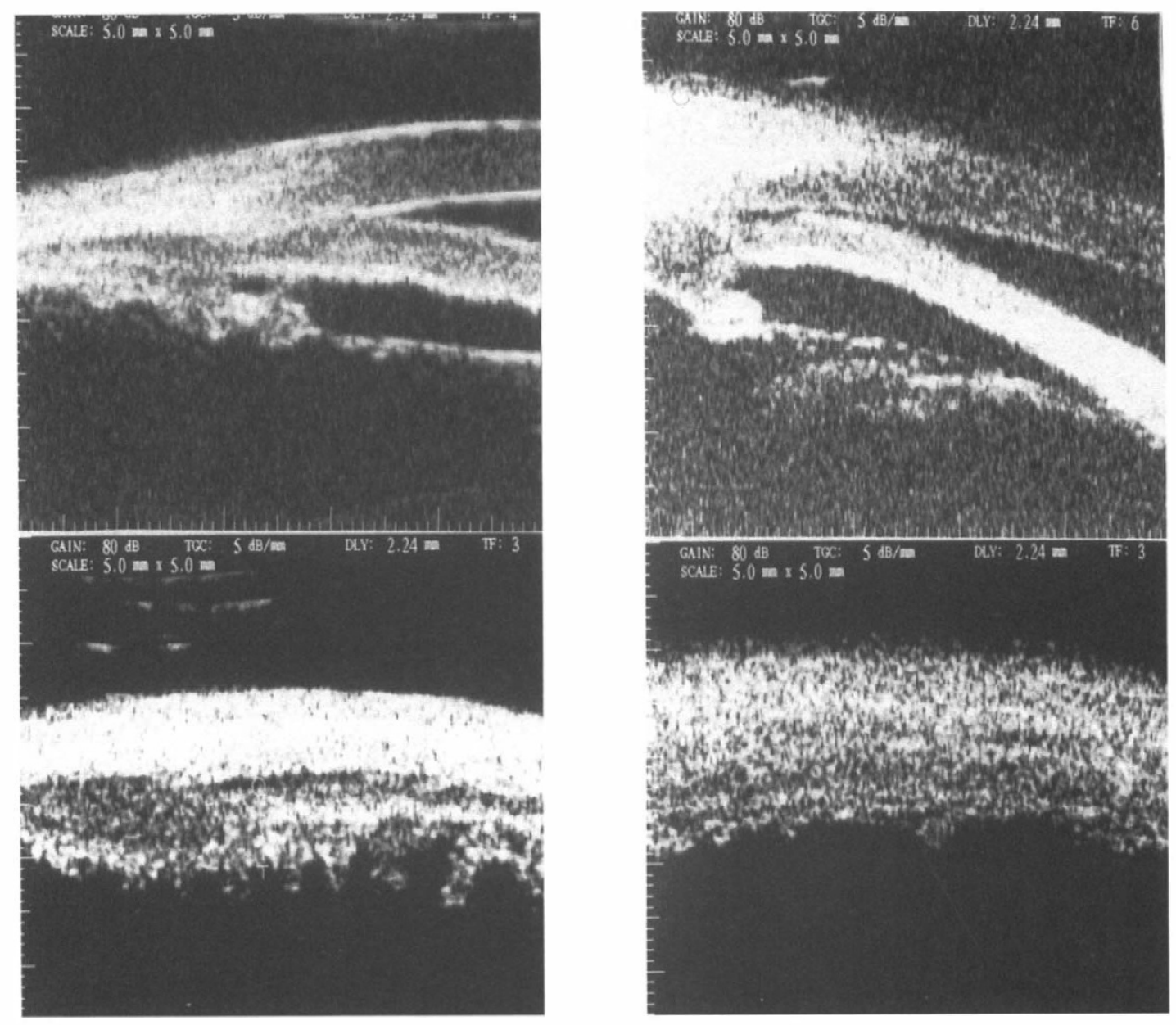

Fig. 3. Hypotony in two patients with cataracts. UBM findings in two sisters with chronic uveitis related to juvenile chronic arthritis of 15 years' duration showed that in one patient hypotony was due to tractional ciliary body detachment (top left) with morphologically normal ciliary villosities (bottom left). In the other patient hypotony was due to ciliary body atrophy (bottom right); note the very shallow anterior chamber with the iris touching the peripheral cornea (top right); cataract surgery was not undertaken in the latter patient because of the high probability of post-operative phthisis.
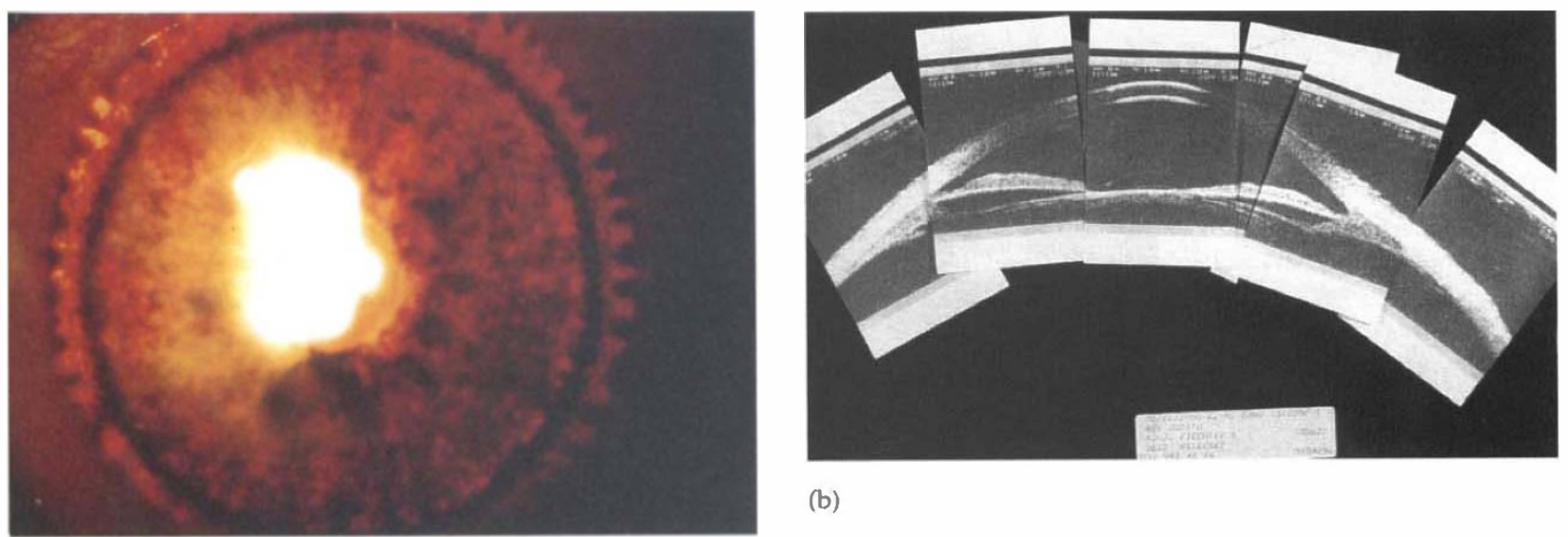

(b)

(a)

Fig. 4. Hypotony in a patient with chronic anterior uveitis due to syphilis. The colour photograph (a) shows iris atrophy especially pronounced at the iris base. The UBM composite picture (b) shows not only thinning of the iris base but also ciliary body atrophy with no villosities identified on the right side of the picture and a thinned ciliary body on the left.

The anatomical location of uveitis was anterior in 17 patients $(22 \%)$, intermediate in $23(30 \%)$ and was a panuveitis in 25 (32\%). In 12 patients (16\%) the pathology was a pseudo-uveitis, mostly post-traumatic inflammation. The list of specific diagnoses or pathologies is shown in Fig. 1.
In Table 1, the proportion of patients in each specific pathological category and the respective quality of information given by the UBM (essential, positive or negative) for the total group of patients and for each subcategory are shown. As can be seen in this table the types of pathologies coming most often to UBM examination were pars plana and iris and ciliary body pathology. 

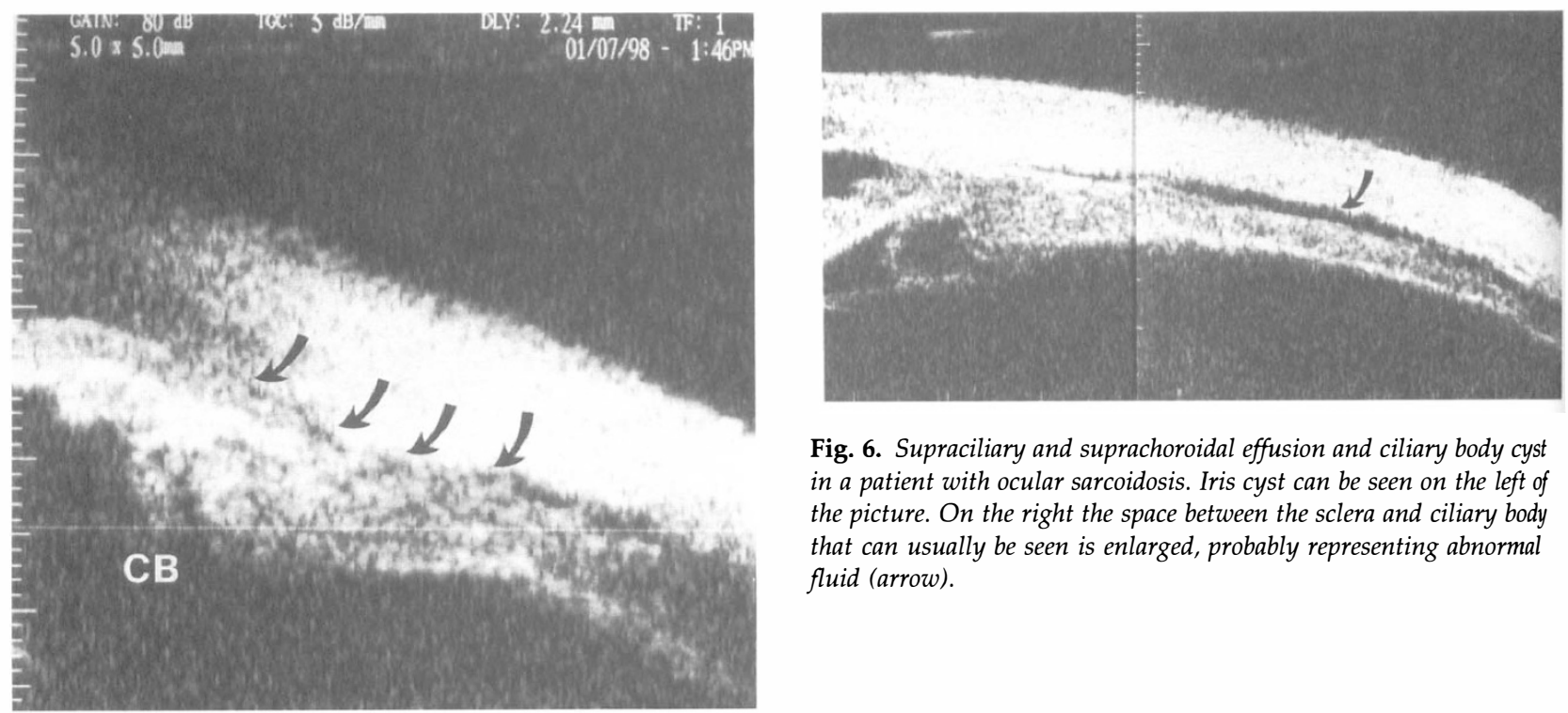

Fig. 6. Supraciliary and suprachoroidal effusion and ciliary body cyst in a patient with ocular sarcoidosis. Iris cyst can be seen on the left of the picture. On the right the space between the sclera and ciliary body that can usually be seen is enlarged, probably representing abnormal fluid (arrow).

Fig. 5. Iridodialysis. This patient was seen in the uveitis unit because of hypotony and persisting inflammation resistant to topical and systemic corticosteroid therapy. History revealed a car accident during which blunt trauma to the eye occurred. UBM revealed an irido- and cyclodialysis (curved arrows). The ciliary body (CB) was not atrophic. Visual function and ocular pressure slowly increased after cyclopexy performed in the area of dialysis.

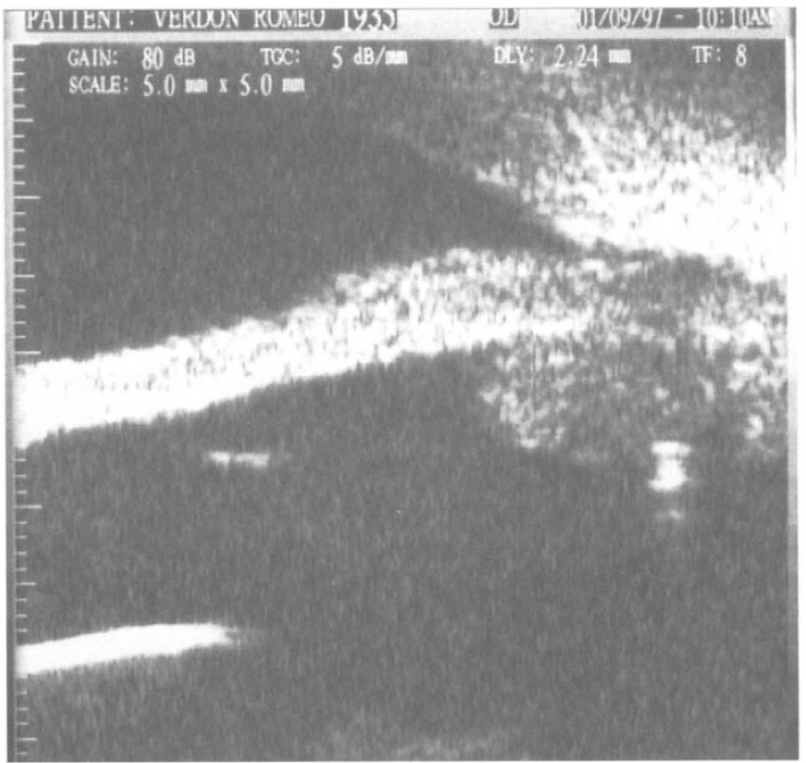

Fig. 7. Haptic of intraocular lens touching the ciliary body in a patient with pseudophakic uveitis. The hyperdense haptic can clearly be identified resting on the ciliary body.

The number of patients in whom UBM contributed significant information amounted to $43 \%$ for the whole group (33/77 patients). The highest yield of results was obtained in the group of patients diagnosed as having Toxocara uveitis. UBM findings in this condition seem to be very specific, showing the typical pseudocystic arrangement of the vitreous (Fig. 2). The second group of patients that benefited from UBM analysis was those with hypotony, for which the yield of significant information was $83 \%$. By showing the cause of hyptony UBM contributed essential information that influenced treatment in 10 of 12 patients. In 4 patients with opaque media following chronic uveitis, UBM showed that hypotony was due to tractional ciliary body detachment by a cyclitic membrane in 2 patients, for whom surgery was indicated (Fig. 3), and due to complete ciliary body atrophy in the other 2 patients, representing a contraindication for cataract surgery because of the danger of phthisis (Fig. 3). In the other cases UBM showed that hypotony was due to ciliary body atrophy ( 3 cases, Fig. 4 ) dialysis of iris and ciliary body (3 cases, Fig. 5), to uveal effusion syndrome in one case (choroidal 


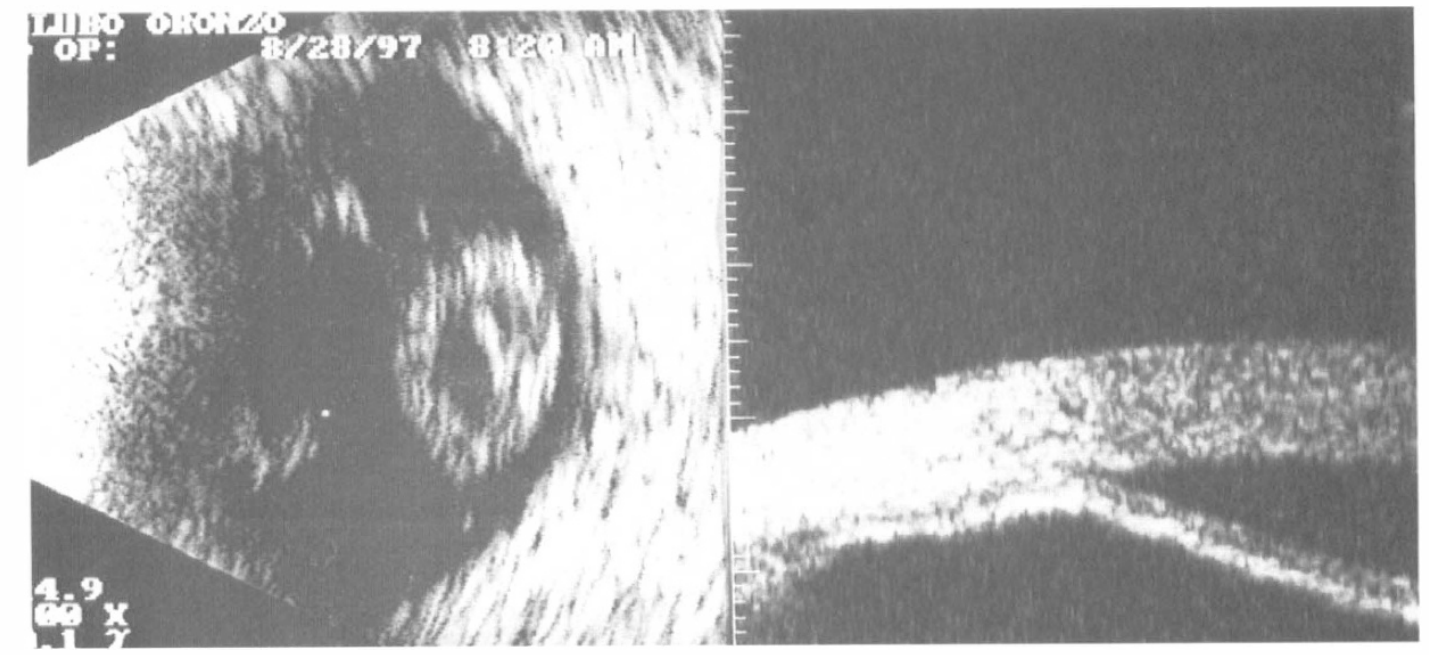

Fig. 8. Ciliary body atrophy in a patient with ocular hypertension. B-scan echography showed a lens luxated into the posterior segment at the origin of phaco-antigenic glaucoma (left). The UBM picture (right) showed complete ciliary body atrophy indicating possible post-surgical phthisis.

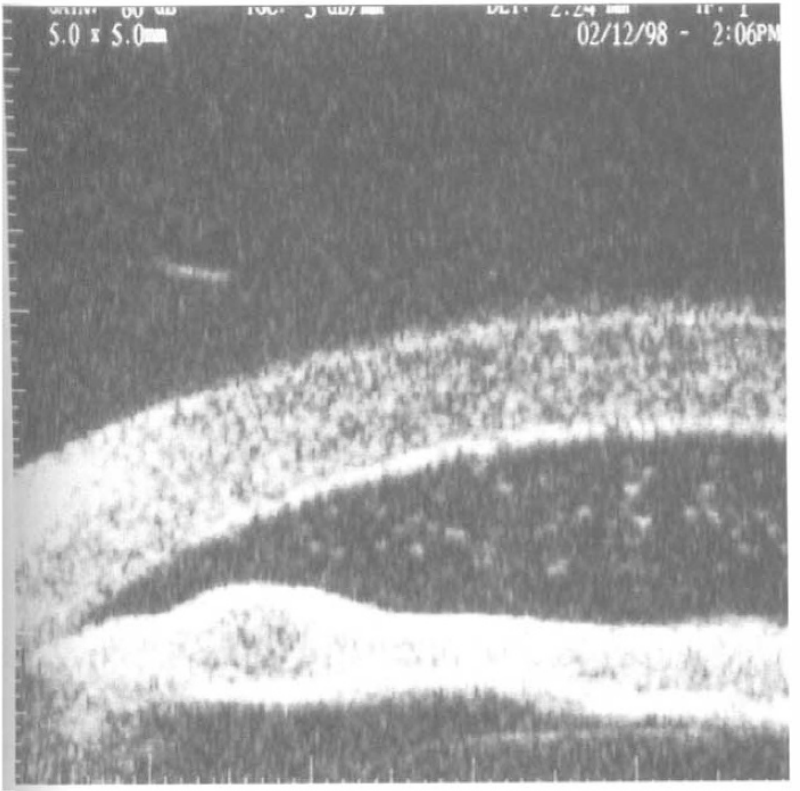

(a)

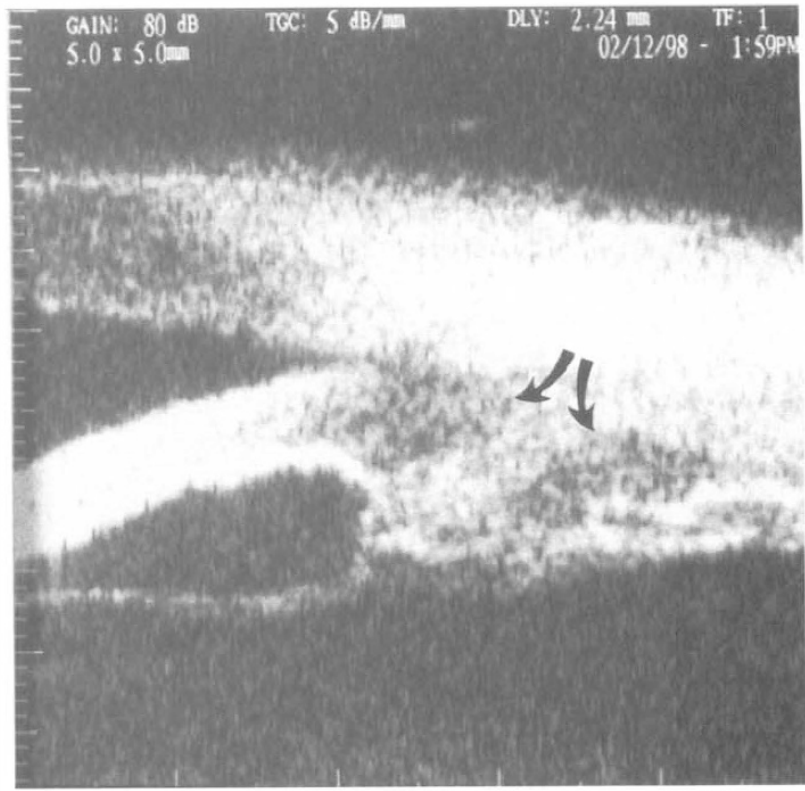

(b)

Fig. 9 Tuberculous granulomas in iris and ciliary body. UBM findings in a patient with chronic granulomatous uveitis show iris nodules with a hyporeflective centre, not only in the iris stroma but also in the ciliary body (arrows), that responded to antituberculous therapy. The patient was not available for post-treatment UBM.

effusion and thickened sclera) and to inflammatory ciliary body detachment in a patient with sarcoidosis (Fig. 6).

UBM was performed in 11 patients with pseudophakic uveitis. It determined the origin of the pathology in all cases and the UBM information was essential in 4 cases $(36 \%)$, showing that inflammation was due to lens fragments in one case versus ciliary body damage in 3 patients for whom the IOL haptics were repositioned surgically (Fig. 7). In all other cases UBM showed that the haptics touched the ciliary body, explaining the intraocular inflammation.

UBM examination performed for iris and ciliary body pathology yielded essential information in 5 of 17 patients (29\%) and findings were positive in 15 of 17 patients. In one case of cataract in a patient with hypertension due to angle closure caused by peripheral anterior synechiae and pupillary seclusion, UBM showed complete atrophy of the ciliary body predicting hypotony as a post-operative complication (Fig. 8). In another case of granulomatous uveitis of possible tuberculous origin, UBM showed multiple nodules with a hypodense centre not only in the iris but also in the ciliary body stroma (Fig. 9). Evolution confirmed the tuberculous aetiology of this uveitis. In 2 cases of pseudo-uveitis, UBM showed an iris and ciliary body metastasis, detected behind a hyphaema in one case (Fig. 10) and a melanoma of the iridocorneal angle in the other. In a patient with Vogt-Koyanagi-Harada syndrome presenting myopisation during a recurrence of inflammation, UBM showed exudative ciliary body detachment. Other positive findings were iris and ciliary 

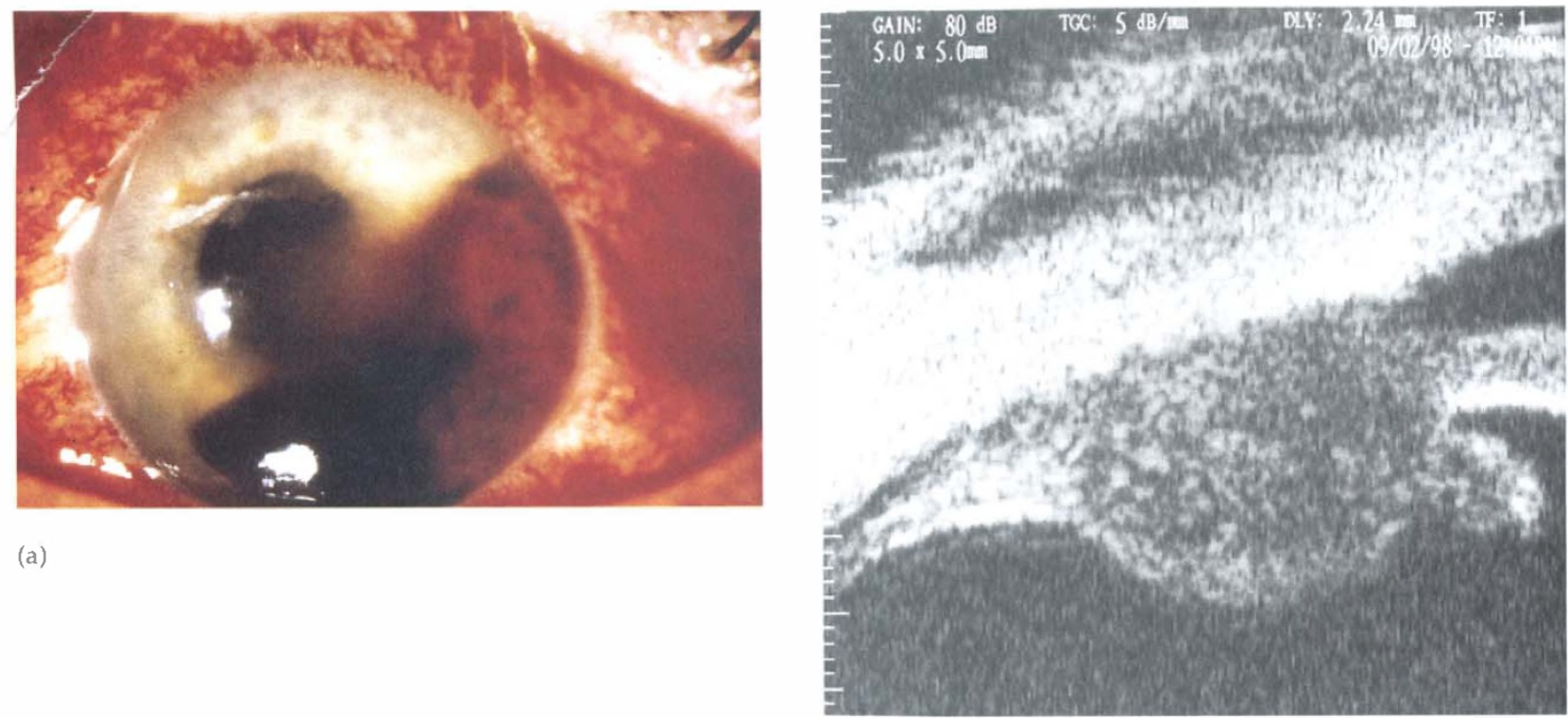

(b)

Fig. 10. Pseudouveitis in a patient with an iris and ciliary body mass. The patient presented with anterior chamber cells and hyphaema (a). The origin of the hyphaema was a ciliary body and iris mass precisely localised by UBM that was later diagnosed as a metastasis of a lung cancer.

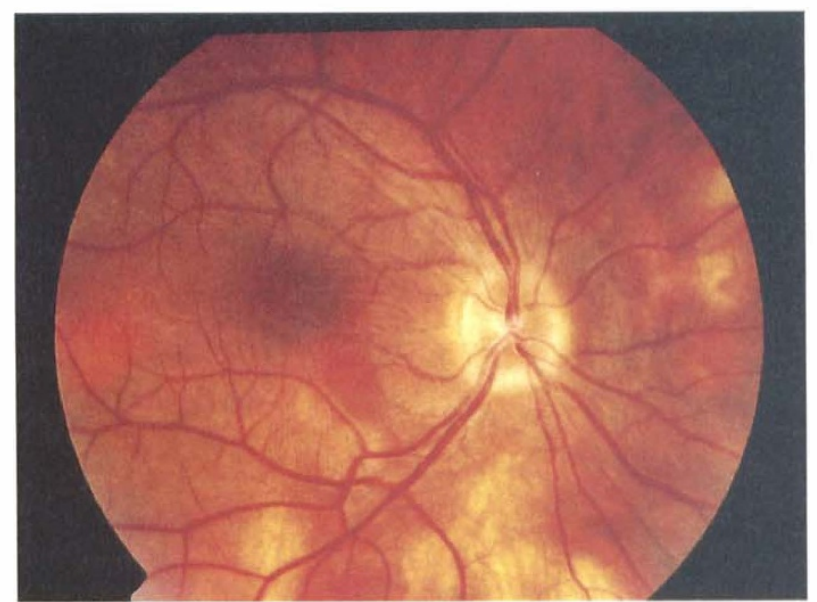

(a)

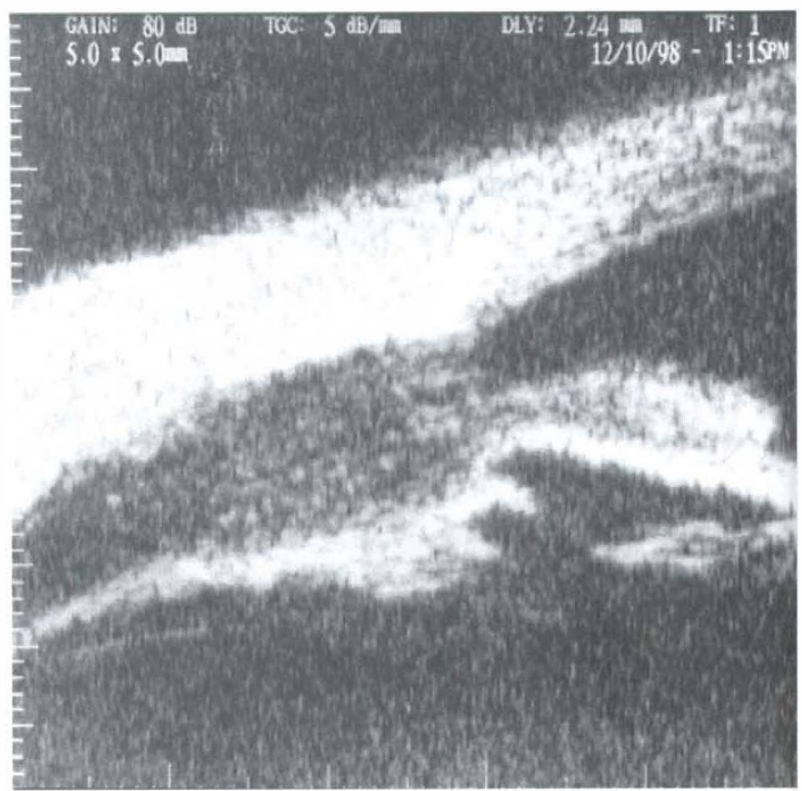

(b)

Fig. 11. Iris and ciliary body dialysis in a hypotonic patient with suspected sarcoid chorioretinitis. The colour photograph shows chorioretinal alterations in a patient with hypotony that remained stationary over 5 years. UBM showed a mass with a bright area between the sclera and ciliary body/iris base, probably secondary to a blunt trauma that was revealed from the patient's history. The mass was suspected to be an organised haemorrhage (b).

body atrophy in 2 patients with herpetic uveitis, ciliary body cysts corresponding to iris bulging in 4 cases and localised fibrosis of the ciliary body in 3 patients with sarcoidosis. In one patient with areas of chorioretinal atrophy and hypotony, UBM showed cyclodialysis caused by mass later found to be due to blunt trauma in the past (Fig. 11).

Pars plana imaging was the indication for UBM examination in 26 of 77 (29\%) of cases. It yielded essential information in 7 of $26(27 \%)$ patients and findings were positive in 23 of 26 patients. In 5 patients diagnosed as having intermediate uveitis ( 2 with opaque media) pars planitis was excluded because of the absence of typical pars plana deposits. Four of these patients were reevaluated and later diagnosed as having Behçet disease in 3 cases (Fig. 12) and sarcoidosis in one patient. In 2 patients diagnosed as having Behçet disease, the presence of typical condensations at the level of the pars plana permitted the diagnosis to be reoriented towards pars planitis (Fig. 13). UBM examination was positive in 14 additional cases showing the presence of typical condensations at the level of the pars plana, confirming 


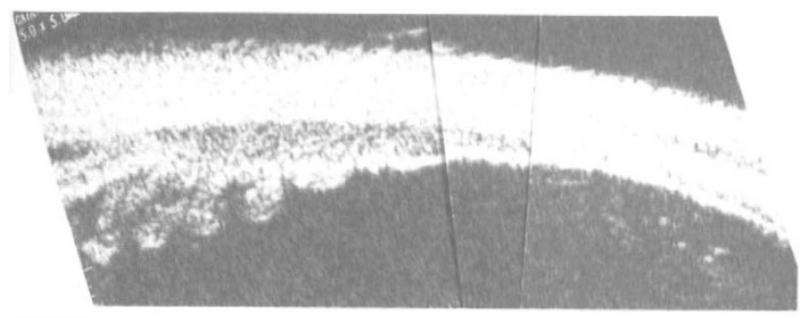

Fig. 12. Pars plana imaging: 1. UBM shows absence of typical pars plana condensations in a patient diagnosed as having pars planitis. Reevaluation of the case led to the diagnosis of Behçet's uveitis.

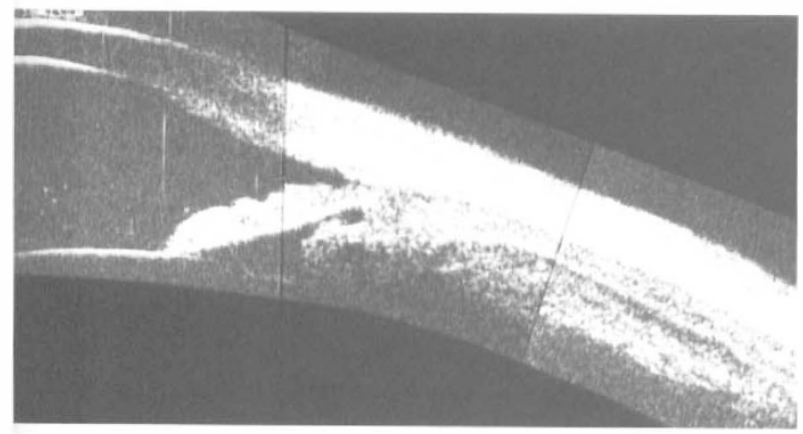

(a)

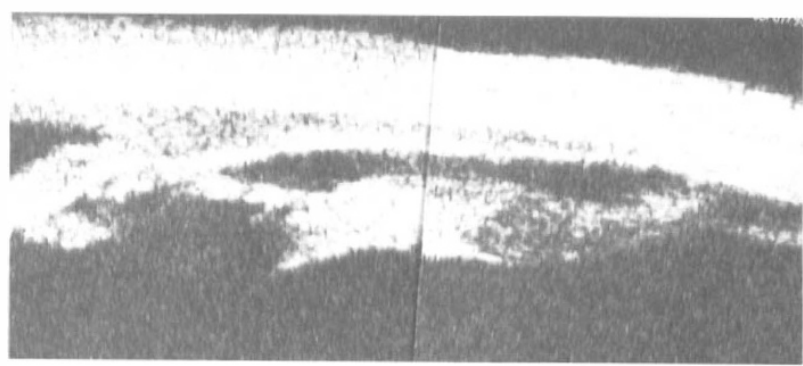

Fig. 13. Pars plana imaging: 2. Presence of typical pars plana condensations in a patient with pronounced retinal vasculitis diagnosed as Behçet's uveitis allowed the diagnosis to be reoriented towards intermediate uveitis of the pars planitis type.

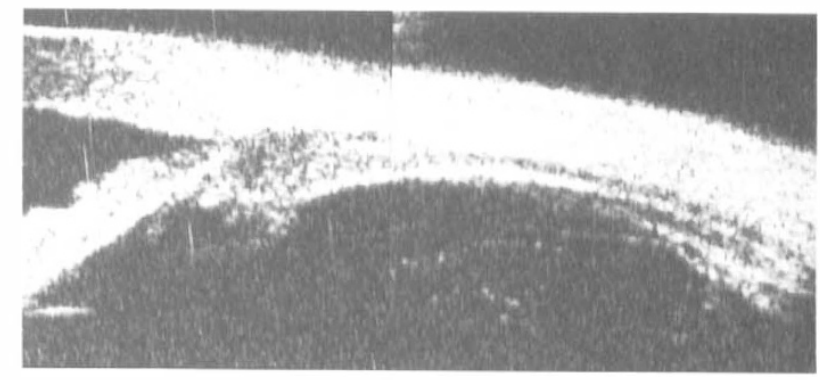

(b)

Fig. 14. Pars plana imaging: 3. Typical UBM pars plana condensations seen in intermediate uveitis of the pars planitis type before treatment (a) that diminished after the introduction of systemic corticosteroids $(b)$.

the diagnosis of pars planitis. In some of these patients therapeutic intervention could be monitored by UBM, showing a reduction of the pars plana condensations (Fig. 14).

Five cases of scleritis were examined by UBM. In all cases UBM examination was positive but it did not contribute essential information. It showed that the inflammatory process involved the whole thickness of the sclera in 3 cases of active disease. In the other 2 cases with scleromalacia it confirmed scleral thinning already shown by the clinical examination.

Among the 77 patients examined, 9 (12\%) had opaque media. UBM examination was especially useful in this group. Findings were essential in all 9 patients as diagnosis or therapeutic attitude relied strongly on the UBM findings. In 2 cases with a very turbid vitreous characteristic pseudocystic transformation of the vitreous allowed the diagnosis of Toxocara uveitis (Fig. 2). In 2 cases with cataracts diagnosed as intermediate uveitis, the absence of typical para plana condensations allowed the diagnosis of paras planitis to be excluded, and reinvestigation later allowed the diagnosis of Behçet's uveitis. In 4 patients with hypotony, UBM identified the cause of hypotony as reported above (Fig. 3). Finally, in a patient with cataract and hypertony due to peripheral anterior synechiae, UBM identified severe atrophy of the ciliary body, reorientating a planned combined glaucoma and cataract operation towards a simple cataract extraction.

\section{Discussion}

The appraisal of uveitis patients has become more precise in recent years following the development of new, mostly non-invasive, investigational methods such as laser flare photometry, ${ }^{7}$ optical coherence tomography ${ }^{8}$ and indocyanine green angiography. ${ }^{7} \mathrm{We}$ evaluated here the use of UBM, one of the new methods, in the investigation of uveitis. The advantage of UBM is that it allows imaging access to structures that cannot be seen otherwise. Indication to perform UBM examination seems to be as high as $8 \%$ in a uveitis clinic. We found that, in a selected group of patients, the yield of essential information that allowed a diagnosis to be reached or determined the therapeutic attitude was over $40 \%$, and the proportion of positive findings was over $90 \%$.

For one entity, Toxocara uveitis, UBM showed specific signs, characterized by pseudocystic degeneration of the vitreous. UBM was found to be very helpful for the diagnosis in patients where Toxocara uveitis was suspected, especially because diagnostic tests available, including Toxocara serology, are of low specificity and sensitivity. The rather high proportion of Toxocara cases in our series is explained by the fact that UBM signs in Toxocara uveitis were described earlier by our group and additional patients with suspected Toxocara uveitis have been sent to us since. ${ }^{10}$

The two groups of patients for which UBM examination was particularly useful were those who presented with opaque media and those with hypotony. The yield of essential information that allowed a diagnosis to be reached or that determined the 
therapeutic attitude was $100 \%$ in the first group and $83 \%$ in the second. In the latter group our experience was in accordance with previous reports stressing the usefulness of UBM in diagnosing the causes of hypotension. ${ }^{11}$

In pseudophakic uveitis UBM was useful in establishing the role of misplaced haptics in the generation of uveitis, confirming the value of UBM in the localisation of the haptics of intraocular lenses. ${ }^{12}$ The assessment of sigificant inflammatory involvement in the iris and ciliary body was achieved by UBM in about $30 \%$ of cases. In accordance with previous reports we found ciliary body cysts in $30 \%$ of these patients, of which Fig. 5 is a typical illustration. ${ }^{13}$ We also documented suprachoroidal effusion in a patient with Vogt-Koyanagi-Harada syndrome as described previously. ${ }^{14}$ Examination of pars plana pathology by UBM has been shown to be especially useful. ${ }^{5,15}$ It allowed us to establish the correct diagnosis in one-third of cases by confirming pars planitis or excluding this diagnosis. In the other cases with positive findings it showed the typical condensations seen in pars planitis that correspond to the snowbanks seen clinically. Followup UBM examinations after the introduction of corticosteroid treatment showed the regression of the pars plana condensations. This indicates that UBM might be useful for the monitoring of therapy, as it is very difficult to evaluate the evolution of the thickness of snowbanks clinically.

We did not find UBM very useful in scleritis. UBM showed the extent of inflammatory involvement without contributing essential information. As indicated in a recently published study, it is probably interesting but not essential to follow cases of anterior scleritis by UBM when available. ${ }^{6}$

UBM was shown to be a valuable additional diagnostic test that contributed to a more precise management of those uveitis cases where the inflammatory process was situated in the anterior uveal structures and vitreous not accessible by clinical examination.

\section{References}

1. Pavlin CJ, Sherar MD, Foster FS. Subsurface ultrasound microscope imaging of the intact eye. Ophthalmology 1990;97:244-50.

2. Pavlin CJ, McWhae JA, McGowan HD, Foster FS. Clinical use of ultrasound biomicroscopy. Ophthalmology 1991;92:287-95.

3. Pavlin CJ, Ritch R, Foster FS. Ultrasound biomicroscopy in the plateau iris syndrome. Am J Ophthalmol 1992;113:390-5.

4. Chiou AGY, Mermoud A, Underdahl JP, Schnyder CC. An ultrasound biomicroscopic study of eyes after deep sclerectomy with collagen implant. Ophthalmology 1998;105:746-50.

5. Gentile RC, Berinstein DM, Liebmann J, Rosen R, Stegman Z, Tello C, Walsh JB, Ritch R. High-resolution ultrasound biomicroscopy of the pars plana and peripheral retina. Ophthalmology 1998;105:478-84.

6. Heiligenhaus A, Schilling M, Lung E, Steuhl KP. Ultrasound biomicroscopy in scleritis. Ophthalmology 1998;105:527-34.

7. Herbort CP, deAncos E, Guex-CRosier Y, Pittet N. Use of laser flare photometry to assess and monitor inflammation in uveitis. Ophthalmology 1997;104:64-72.

8. Sourdille P, Santiago PY. Optical coherence tomography of macular thickness after cataract surgery. J Cataract Refract Surg 1999;25:256-61.

9. Herbort CP, LeHoang P, Guex-Crosier Y. Schematic indocyanine angiographic protocol for the evaluation of posterior uveitis. Ophthalmology 1998;105:432-40.

10. Tran VT, Lumbroso L, LeHoang P, Herbort CP. Ultrasound biomicroscopy in peripheral retinovitreal toxocariasis. Am J Ophthalmol 1999;127:607-9.

11. Minamoto A, Nakano KE, Tanimoto S, Mizote H, Takeda Y. Ultrasound biomicroscopy in the diagnosis of persistent hypotony after vitrectomy. Am J Ophthalmol 1997;123:711-3.

12. Pavlin CJ, Rootman D, Arshinoff S, et al. Determination of haptic position of transsclerally fixated posterior chamber intraocular lenses by ultrasound biomicroscopy. J Cataract Refract Surg 1993;19:573-7.

13. Gentile RC, Liebmann JM, Tello C, Stegman Z, Weissmann SS, Ritch R. Ciliary body enlargement and cyst formation in uveitis. Br J Ophthalmol 1996;80:895-9.

14. Kawano YI, Tawara A, Nishioka Y, Suyama Y, Sakamoto H, Inomata $\mathrm{H}$. Ultrasound biomicroscopic analysis of transient shallow anterior chamber in Vogt-Koyanagi-Harada syndrome. Am J Ophthalmol 1996;121:720-3.

15. Garcia-Feijoo J, MartinCarbajo M, Benitez del Castillo JM, Garcia-Sanchez J. Ultrasound biomicroscopy in pars planitis. Am J Ophthalmol 1996;121:214-5. 